\title{
Rotational spectrum of tryptophan
}

\author{
M. Eugenia Sanz, ${ }^{\text {a) }}$ Carlos Cabezas, ${ }^{\text {b) }}$ Santiago Mata, ${ }^{\text {c) }}$ and Josè L. Alonso ${ }^{\text {d) }}$ \\ Grupo de Espectroscopia Molecular (GEM), Edificio Quifima, Laboratorios de Espectroscopia y \\ Bioespectroscopia, Unidad Asociada CSIC, Parque Científico Uva, Universidad de Valladolid, \\ 47011 Valladolid, Spain
}

\begin{abstract}
The rotational spectrum of the natural amino acid tryptophan has been observed for the first time using a combination of laser ablation, molecular beams, and Fourier transform microwave spectroscopy. Independent analysis of the rotational spectra of individual conformers has conducted to a definitive identification of two different conformers of tryptophan, with one of the observed conformers never reported before. The analysis of the ${ }^{14} \mathrm{~N}$ nuclear quadrupole coupling constants is of particular significance since it allows discrimination between structures, thus providing structural information on the orientation of the amino group. Both observed conformers are stabilized by an $\mathrm{O}-\mathrm{H} \cdot \cdots \mathrm{N}$ hydrogen bond in the side chain and a $\mathrm{N}-\mathrm{H} \cdot \cdots \pi$ interaction forming a chain that reinforce the strength of hydrogen bonds through cooperative effects.
\end{abstract}

\section{INTRODUCTION}

The spectroscopic investigation of biologically relevant molecules in the gas phase has flourished over the last decades. The application of techniques such as laser desorption/ablation, Matrix-Assisted Laser Desorption/Ionization

(MALDI), and electrospray ionization (ESI) had made possible the successful vaporization of biomolecules as neutrals and ions from solids and solutions and their analysis using mass spectrometry in combination with a variety of methods. ${ }^{1-5}$ "Action" spectra and double-resonance techniques are in widespread use to detect different conformations of biomolecules, and, in some cases, identify their structures. ${ }^{6-10}$ Because the majority of these techniques use multiphoton resonantly enhanced ionization, they have been applied to molecules containing UV chromophores. Rotational spectroscopy, considered as the most powerful technique for structural characterisation, is not constrained by the need of a chromophore, but it had somehow lagged behind other gas-phase spectroscopic methods in the study of solid biomolecules. First accounts of laser ablation in combination with rotational spectroscopy were published in the $1990 \mathrm{~s}^{11,12}$ but further development and optimization did not occur until the first years of the 21 st century, ${ }^{13}$ opening up a new range of molecules for rotational studies. Initial investigations were

devoted to aliphatic amino acids, since no previous conformational data were available on them, with the exceptions of glycine ${ }^{11,14}$ and alanine. ${ }^{15}$ To date, detailed and unambiguous conformational information has been reported on almost all of the proteinogenic amino acids using this Laser

\footnotetext{
${ }^{a)}$ Present address: Department of Chemistry, King's College London, London SE11DB, United Kingdom. Electronic mail: maria.sanz@kcl.ac.uk. Telephone: $+44(0) 2078487509$.

b)E-mail: ccabezas@qf.uva.es. Telephone: +34 983186348.

c) E-mail: santiago.mata@uva.es. Telephone: +34 983186349.

d) Author to whom correspondence should be addressed. Electronic mail: jlalonso@qf.uva.es. Telephone: +34 983186345. Fax: +34 983186349.
}

Ablation Molecular Beam Fourier Transform Microwave (LA-MB-FTMW) spectroscopy.

To test the applicability of this experimental method to aromatic molecules, we firşt studied the aromatic unnatural amino acid phenylglycine. The $532 \mathrm{~nm}$ green light of a nanosecond Nd:YAG laser was used for vaporization. A very intense rotational spectrum was observed and two conformers of phenylglycine were identified. However, when the sames experimental setup was used in the study of phenylalanine, the observed microwave spectrum was notably weak. The situation was reproduced for tyrosine and more acutely for tryptophan (Trp). To understand the processes that were taking place in the ablation plume, we placed the laser ablation nozzle used in our microwave experiment into a bespoke time-of-flight mass spectrometer and interrogated aromatic amino acids. Significant photofragmentation was detected and consequently, our experiment was modified trying to minimize it. Several experimental parameters were changed, of which the most important were the length of the laser pulse and the wavelength of the laser light. Shorter (picosecond) laser pulses and shorter laser wavelengths ( $355 \mathrm{~nm}$ ) were found to improve the intensity of rotational spectra considerably, which allowed the identification of several conformers of phenylalanine. In this paper, we present the first rotational spectrum of the aromatic amino acid tryptophan.

Tryptophan has been extensively studied by several research groups using different flavours of laser spectroscopy in supersonic expansions. The pioneering experiment of Levy $^{3}$ measured the resonantly-enhanced two-photon ionization (REMPI) spectrum of Trp and observed six different conformers, although no structural identifications were made. Piuzzi et al. observed three of those conformers and confirm that they were distinct, using REMPI and holeburning spectroscopies. ${ }^{22}$ Simons and co-workers characterized six species through UV hole-burning and infrared iondip spectroscopy and were able to match five of them to 
<smiles>N[C@H](Cc1c[nH]c2ccccc12)C(=O)O</smiles>

SCHEME 1. Schematic representation of Trp.

specific conformational structures. ${ }^{23}$ Using IR action spectroscopy, Bakker et al. ${ }^{24}$ observed three different conformers. Recently, a cavity ring-down absorption experiment observed seven tryptophan conformers, adding a new one to the six previously reported. ${ }^{25}$ No reports on the rotational spectrum of tryptophan had been published up to now.

Trp is an indole derivative with an alanyl side chain. It possesses two nitrogen atoms, in the amino group $\left(\mathrm{N}_{\mathrm{a}}\right)$ and in the pyrrole ring $\left(\mathrm{N}_{\mathrm{i}}\right)$ (Scheme 1). The most abundant isotope of nitrogen, ${ }^{14} \mathrm{~N}$, has a nuclear quadrupole moment $I$ $=1$, which interacts with the electric field gradient created by the rest of the molecule at the nucleus. This is of particular relevance for rotational spectroscopy, as this technique has an inherently high resolution that not only allows the simultaneous detection and analysis of several conformers of an amino acid but also permits the observation of hyperfine effects. The ${ }^{14} \mathrm{~N}$ nuclear quadrupole coupling splits the rotational energy levels and gives rise to the appearance of a hyperfine structure in each transition of the rotational spectrum. The hyperfine pattern depends strongly on the local environment of the ${ }^{14} \mathrm{~N}$ quadrupolar nucleus, including changes in the orientation of the amino group. It is therefore a precious tool in conformational identification by rotational spectroscopy. Through the analysis of the rotational spectrum of Trp, we will show how nuclear quadrupole coupling acts as a reporter of the interactions involving $\mathrm{N}_{\mathrm{a}}$ and $\mathrm{N}_{\mathrm{i}}$ quadrupolar nuclei.

The difficulty of observing the rotational spectrum of Trp was compounded by two factors besides significant photofragmentation. Quadrupole coupling produced by two ${ }^{14} \mathrm{~N}$ nuclei splits each rotational level in several sublevels, and consequently, the overall intensity of each rotational transition is spread over many hyperfine components. In addition, Trp is the largest of the proteinogenic amino acids. It has smaller rotational constants that shift its rotational spectrum towards lower frequencies, out of the usual frequency range of operation of molecular beam Fourier transform microwave spectrometers $(5-18 \mathrm{GHz})$. A recently constructed LA-MBFTMW spectrometer, specifically designed to optimize detection of heavier molecules, at lower frequency range, has been used for Trp. The enhanced sensitivity achieved with the new instrument, along with improvements in laser ablation allowed the observation and structural characterization of two different conformers of Trp, one of them not previously reported. Details of the investigation are given in Secs. II A and II B.

\section{METHODS}

\section{A. Experimental}

The rotational spectrum of Trp was observed using a new LA-MB-FTMW spectrometer operating in the $2-10 \mathrm{GHz}$ frequency range. ${ }^{26}$ Solid samples of L-tryptophan (m.p. $\left.\sim 280{ }^{\circ} \mathrm{C}\right)$ and isotopically enriched $\left({ }^{15} \mathrm{~N}_{\mathrm{i}}\right.$ and $\left.{ }^{15} \mathrm{~N}_{\mathrm{i}}{ }^{15} \mathrm{~N}_{\mathrm{a}}\right)$ DLtryptophan were used. The amino acid samples were mixed with a few drops of a commercial binder, introduced into a cast and converted into cylindrical rods after being subjected to a few tons of pressure. Initially, the second harmonic at $532 \mathrm{~nm}$ of a nanosecond Nd:YAG laser was employed with energies of ca. $50 \mathrm{~mJ} /$ pulse for vaporization. This resulted in the observation and assignment of one conformer of tryptophan, but the rotational spectrum was extremely weak, with signal-to-noise ratios for the most intense transitions of $\mathrm{S} / \mathrm{N}$ $=3$ after accumulating 500 spectra. Tests were carried out with an assortment of binders and adding UV absorbers to the sample, but improvements in spectral signal were marginal. Changing to the third harmonic at $355 \mathrm{~nm}$ produced a small increase in intensity with $\mathrm{S} / \mathrm{N}=4$ after 500 cycles. Afterwards, two new Nd:YAG lasers capable of producing shorter laser pulses of $150 \mathrm{ps}$ and $35 \mathrm{ps}$ at $355 \mathrm{~nm}$ were tried. Moving from a $5 \mathrm{~ns}$ to a $150 \mathrm{ps}$ laser pulse returned $\mathrm{S} / \mathrm{N}=6$, again with 500 cycles. A further decrease of the laser pulse length by a factor of 5 , to $35 \mathrm{ps}$, yielded $\mathrm{S} / \mathrm{N}=20$ with 250 cycles, the most dramatic change. The optimal laser power used for vaporization also changed, falling to $1-5 \mathrm{~mJ} /$ pulse for the 35 ps laser. This produced less observable damage in the surface of the rod and decreased sample consumption, a major concern when using expensive isotopically enriched samples.

Once Trp molecules are in the gas phase, they are conducted to the Fabry-Pérot resonator using $\mathrm{Ne}$ as carrier gas at pressures of 15 bar. Microwave radiation pulses of $0.3 \mu$ s polarized tryptophan molecules. After a suitable delay to allow for the extinction of the polarising radiation in the vacuum chamber, the molecular de-excitation signals were collected in the time domain and Fourier-transformed to obtain the frequency domain spectrum. In our setup, microwave radiation travels parallel to the axis of the Fabry-Pérot resonator and therefore all transitions appear as doublets due to the Doppler effect. The molecular frequency is calculated as the arithmetic mean of the Doppler doublets. The accuracy of the frequency measurements is estimated to be better than $3 \mathrm{kHz}$.

\section{B. Computational}

$A b$ initio calculations were carried out to search for the conformational minima of Trp in the potential energy surface and to determine their relative energies. To generate the initial geometries the different types of hydrogen bonds that can be established between the amino and carboxylic groups, $\mathrm{N}-\mathrm{H} \cdot \cdots \mathrm{O}=\mathrm{C}$ (type I), $\mathrm{O}-\mathrm{H} \cdot \cdots \mathrm{N}$ (type II), and $\mathrm{N}-\mathrm{H} \cdots$ $\mathrm{O}-\mathrm{H}$ (type III), were considered. We also considered the different orientations of the side chain, labelled according to the approximate values of the dihedral angle $\_1 \mathrm{C}_{\mathrm{COOH}} \mathrm{C}_{\alpha} \mathrm{C}_{\beta} \mathrm{C}_{\gamma}$ as

a $\left(+60^{\circ}\right), \mathbf{b}\left(-60^{\circ}\right)$, and $\mathbf{c}\left(180^{\circ}\right)$. In addition, conformations arising from the different positions of the indole ring were 

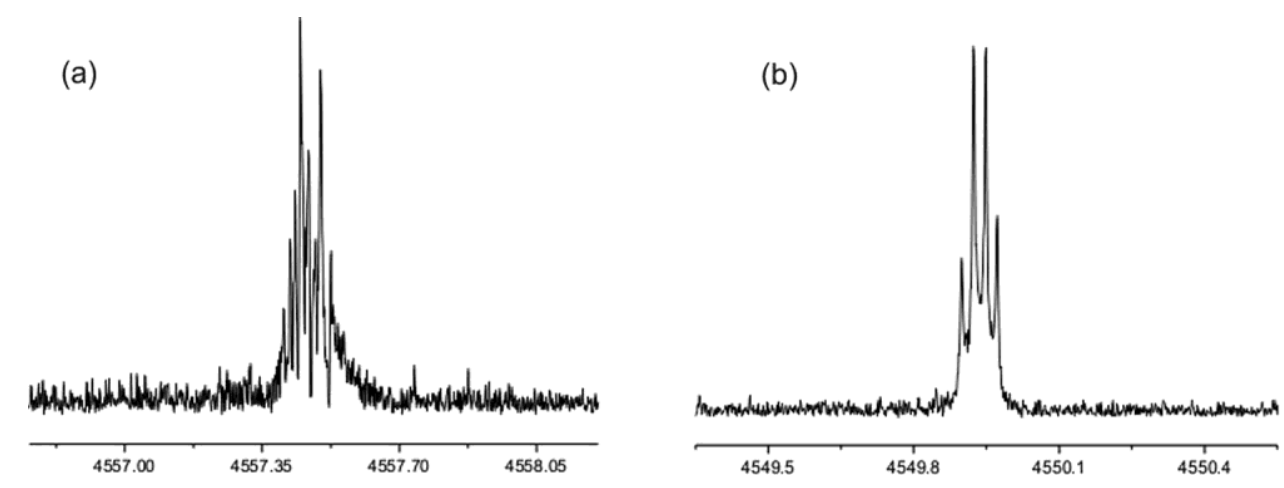

FIG. 1. The 6 615-514 rotational transition for rotamer I of Trp showing the very complex nuclear quadrupole hyperfine structure of the ${ }^{14} \mathrm{~N}_{\mathrm{i}}{ }^{14} \mathrm{Na}_{\mathrm{a}}$ isotopic species (a) and showing the well resolved hyperfine structure for ${ }^{15} \mathrm{Ni}^{-}{ }^{14} \mathrm{Na}$ isotopic species (b). The coaxial arrangement of the adiabatic expansion and the resonator axis produces an instrumental Doppler doubling. The resonant frequencies are calculated as the average of the two Doppler components.

also taken into account. These are defined by the dihedral angle $1 \mathrm{C}_{\alpha} \mathrm{C}_{\beta} \mathrm{C}_{\gamma} \mathrm{C}$, which can adopt values of $\mathrm{ca}$. $+90^{\circ}$ or

$-90^{\circ}$ corresponding to arrangements where the amino group is closer to the pyrrole ring of indole $(+)$ or to the benzene ring $(-)$, respectively. Therefore, three indexes are used to tag the possible conformers of Trp. The B3LYP hybrid functional with the 6-311++G(d,p) basis set was used to optimize geometries and to obtain corrections to the zero-point energy and Gibbs free energies while verifying that all structures were real minima. MP2/6-311++G(d,p) structure optimizations were performed on the B3LYP optimized geometries, as calculations at this level have been found to be optimal for amino acids. Frequency calculations were not carried out at MP2 level as they are too computationally expensive for a system of the size of Trp. The 17 lowest-energy conformations found with this procedure are shown in Figure S1 of the supplementary material ${ }^{37}$ together with their spectroscopic parameters relevant for conformational identification by rotational spectroscopy (Table $\mathrm{S} 1$ of the supplementary material ${ }^{37}$ ), namely, the rotational and quadrupole coupling constants, and the dipole moment components along the principal inertial axes. The conformational minima obtained are in agreement with those previously reported. ${ }^{27,28}$

\section{RESULTS AND DISCUSSION}

Wide frequency scans using different experimental conditions lead to the identification of a set of weak $\mu_{\mathrm{a}}$-type $\mathrm{R}$ branch transitions, showing the $B+C$ separation typical of tops close to the prolate limit. All low-energy conformers of Trp are predicted to be near prolate asymmetric rotors. After assignment of the a-type transitions, new predictions and observations allowed the assignment of $\mu_{\mathrm{b}}$-type R-branch lines. As it can be anticipated, all observed transitions appeared split into many close hyperfine components, arising from the nuclear quadrupole coupling interactions of two quadrupolar nuclei ${ }^{14} \mathrm{~N}_{\mathrm{a}}$ and ${ }^{14} \mathrm{~N}_{\mathrm{i}}$. Figure 1(a) shows the hyperfine structure for the $6_{15} \leftarrow 5_{14}$ rotational transition with many quadrupole components within a small range of frequency, which makes the analysis very complicated. In a first approach, only the frequency centre of each rotational transition was considered in a rigid rotor fit to obtain a preliminary set of rotational constants
$A \approx 1243.4 \mathrm{MHz}, B \approx 392.5 \mathrm{MHz}$, and $C \approx 346.9 \mathrm{MHz}$. Using the same strategy adopted in other studies of amino acids, ${ }^{16-20}$ these values were compared with those predicted for the 17 lowest energy conformers, listed in Table S1 of the supplementary material. ${ }^{37}$ They were found to be compatible with those predicted for the global minimum IIb+ and also with those for conformers $\mathbf{I a -}$ and $\mathbf{I b +}$, collected in Table I. These species present similar mass distribution, with comparable values of the rotational constants. Although the observation $\mu_{\mathrm{a}}$ - and $\mu_{\mathrm{b}}$-type spectra points to the presence of conformer IIb+ in our supersonic expansion (see predicted dipole moment components in Table I), a definitive discrimination between the conformers solely based on rotational constants becomes unattainable.

A close look at the predicted values of the quadrupole coupling constants $\left(\chi_{a a}, \chi_{b b}\right.$, and $\left.\chi_{c c}\right)$ for conformers IIb+, Ia-, and IIa- clearly indicates that they constitute a unique tool in the conformational ascription. ${ }^{29}$ While the predicted values of the quadrupole constants do not change significantly for the indolic ${ }^{14} \mathrm{~N}_{\mathrm{i}}$ nucleus, which is consistent with the similar electronic environment of the ${ }^{14} \mathrm{~N}_{\mathrm{i}}$ in all these conformers, the different arrangement of the $\mathrm{NH}_{2}$ group in the side chains gives rise to large differences for the predicted quadrupole constants for the ${ }^{14} \mathrm{~N}_{\mathrm{a}}$ nucleus. Taking this into account a sample of enriched ${ }^{15} \mathrm{~N}_{\mathrm{i}^{-}}{ }_{14}^{14} \mathrm{~N}_{\mathrm{a}}$ was used to determine the experimental values of the $\mathrm{N}_{\mathrm{a}}$ quadrupole coupling constants. Since ${ }^{15} \mathrm{~N}$ has no quadrupole moment, only the quadrupole hyperfine structure arising from the ${ }^{14} \mathrm{~N}$ in the amino group will appear in the spectrum. The same set of $\mu_{\mathrm{a}}$-type R-branch transitions detected for the parent ${ }^{14} \mathrm{~N}_{\mathrm{i}^{-}}{ }^{14} \mathrm{~N}_{\mathrm{a}}$ species was readily located and assigned. All the transitions exhibited now fully resolved nuclear quadrupole coupling hyperfine structure (see the $6_{15} \leftarrow 5_{14}$ rotational transition in Figure 1(b)) corresponding to the presence of a single ${ }^{14} \mathrm{~N}_{\mathrm{a}}$ nucleus in the observed species. A total of 34 hyperfine components (Table S3 of the supplementary material ${ }^{37}$ ) were fitted ${ }^{30}$ using the Watson's semirigid rotor Hamiltonian in the A reduction and the $\mathrm{I}^{\mathrm{r}}$ representation, $H_{\mathrm{R}}{ }^{(\mathrm{A})}{ }^{31}$ supplemented with an additional term $H_{\mathrm{Q}}{ }^{32}$ to consider the quadrupole coupling interaction. The coupling scheme $I+J=F$ was used. The determined rotational and quadrupole coupling constants are listed in the second column of Table II. Despite the fact that the value of $\chi_{\text {aa }}$ is not well determined from the fit and was thus kept fixed 
TABLE I. Calculated ${ }^{\mathrm{a}}$ spectroscopic parameters for the IIb+, Ia-, and IIa- conformers of Trp (extracted from Table S1 of the supplementary material ${ }^{37}$ ).

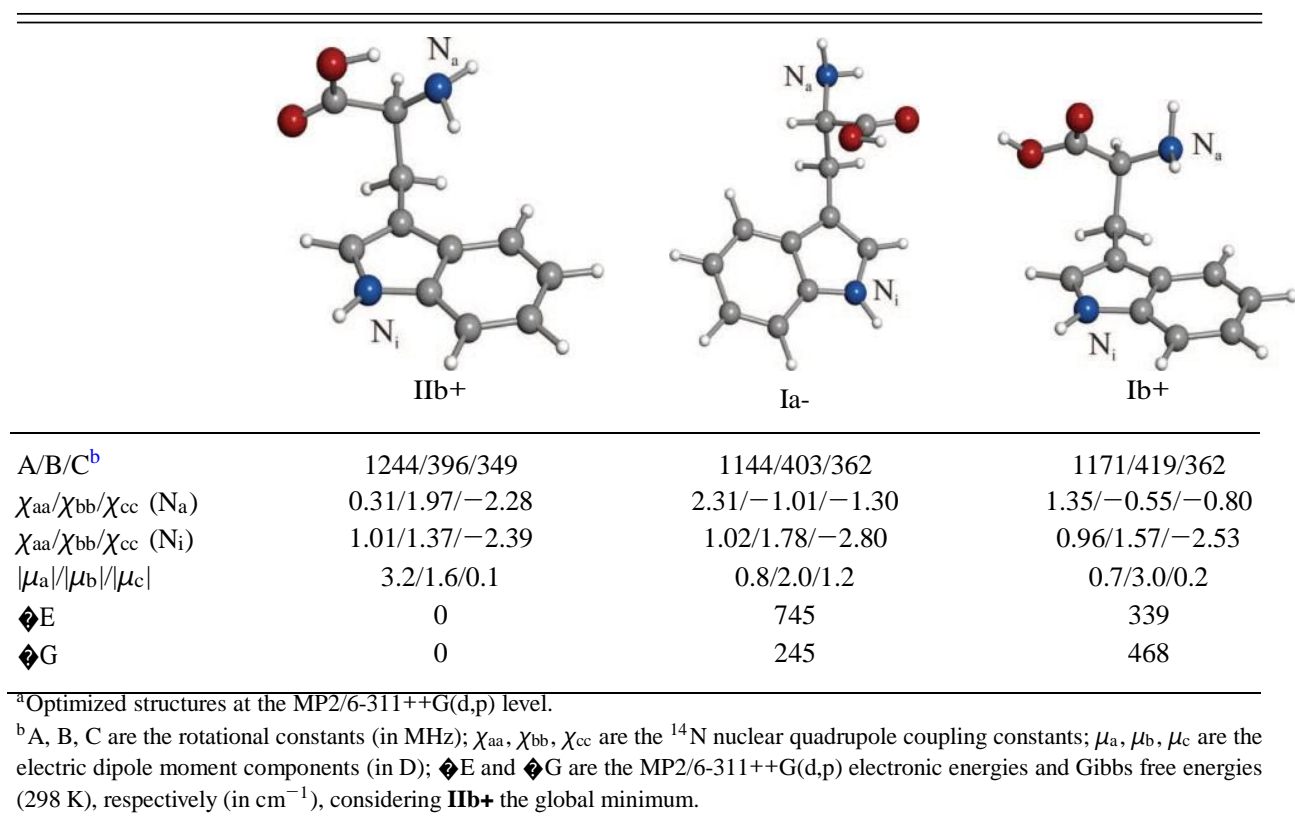

to $a b$ initio value, the comparison between the experimental and theoretical values of $\chi_{\mathrm{bb}}$ and $\chi_{\mathrm{cc}}$ for the amino ${ }^{14} \mathrm{~N}_{\mathrm{a}}$ allow the unambiguous identification of the observed rotamer as conformer as IIb+.

Once the analysis of ${ }^{15} \mathrm{~N}_{\mathrm{i}^{-}}{ }^{14} \mathrm{~N}_{\mathrm{a}}$ was accomplished for the IIb+ conformer, the hyperfine structure for the ${ }^{14} \mathrm{~N}_{\mathrm{i}^{-}}{ }^{14} \mathrm{~N}_{\mathrm{a}}$ isotopic species was analysed using the previously obtained values of the nuclear quadrupole coupling constants for ${ }^{14} \mathrm{~N}_{\mathrm{a}}$ nucleus. Hence, the hyperfine structure was totally assigned and 45 hyperfine components (Table S2 of the supplementary material ${ }^{37}$ ) were fitted to Watson's semirigid rotor Hamiltonian, $H_{\mathrm{R}}{ }^{(\mathrm{A})}$, including a term $H_{\mathrm{Q}}$ to account for the quadrupole interactions of two quadrupolar nuclei. ${ }^{32} H_{\mathrm{Q}}$ was set up in the

TABLE II. Rotational parameters for the observed rotamer I of Trp.

\begin{tabular}{|c|c|c|c|}
\hline & ${ }^{14} \mathrm{~N}_{\mathrm{i}-}{ }^{14} \mathrm{~N}_{\mathrm{a}}$ & ${ }^{15} \mathrm{~N}_{\mathrm{i}-}{ }^{14} \mathrm{~N}_{\mathrm{a}}$ & ${ }^{15} \mathrm{~N}_{\mathrm{i}^{-}}{ }^{15} \mathrm{~N}_{\mathrm{a}}$ \\
\hline $\mathrm{A}^{\mathrm{a}}$ & $1243.5844(29)^{b}$ & $1231.0742(24)$ & $1219.484(7)$ \\
\hline B & $392.48409(11)$ & $392.15526(17)$ & $391.33142(8)$ \\
\hline $\mathrm{C}$ & $346.88467(16)$ & $345.65584(22)$ & 344.31779 (9) \\
\hline$\hat{\mathrm{J}}_{\mathrm{J}}$ & $0.03329(85)$ & $0.0350(15)$ & 0.03205 \\
\hline \multicolumn{4}{|l|}{$\mathrm{N}_{\mathrm{a}}$} \\
\hline$\chi_{\text {aа }}$ & {$[0.31]^{\mathrm{c}}$} & {$[0.31]^{\mathrm{c}}$} & $\ldots$ \\
\hline$\chi_{\mathrm{bb}}$ & 1.714 & 1.777 & $\ldots$ \\
\hline$\chi_{\mathrm{cc}}$ & -2.024 & -2.087 & $\ldots$ \\
\hline$\chi_{\mathrm{bb}-}-\chi_{\mathrm{cc}}$ & $3.739(41)$ & 3.865 (17) & $\ldots$ \\
\hline \multicolumn{4}{|l|}{$\mathrm{N}_{\mathrm{i}}$} \\
\hline$\chi_{\text {aa }}$ & $1.079(74)$ & $\ldots$ & $\ldots$ \\
\hline$\chi_{\mathrm{bb}}$ & $1.301(55)$ & $\ldots$ & $\ldots$ \\
\hline$\chi_{\mathrm{cc}}$ & $-2.380(55)$ & $\ldots$ & $\ldots$ \\
\hline $\mathrm{N}$ & 45 & 34 & 10 \\
\hline$\sigma$ & 2.8 & 2.9 & 0.8 \\
\hline
\end{tabular}

${ }^{\mathrm{a}} \mathrm{A}, \mathrm{B}, \mathrm{C}$ are the rotational constants (in $\mathrm{MHz}$ ); ${ }_{\mathrm{J}}$ is the quartic centrifugal distortion constant (in $\mathrm{kHz}$ ); $\chi_{\mathrm{aa}}, \chi_{\mathrm{bb}}, \chi_{\mathrm{cc}}$ are the ${ }^{14} \mathrm{~N}$ nuclear quadrupole coupling constants (in $\mathrm{MHz}$ ); $\mathrm{N}$ is the number of fitted transitions; $\sigma$ is the rms deviation of the fit (in kHz).

${ }^{\mathrm{b}} \mathrm{S}$ tandard error in parentheses in units of the last digit.

${ }^{\mathrm{c}}$ Parameter kept fixed in the fit. coupled basis set $\left(I_{1} I_{2} I J F\right), I_{1}+I_{2}=I, I+J=F$. The quantum numbers $J, K_{-1}, K_{+1}, I, F$ are used to label the energy levels involved in each transition. The parameters determined from the fit are the rotational constants $A, B, C$ and the diagonal constants of the quadrupole coupling tensor $\chi_{\text {aa }}$, $\chi_{\mathrm{bb}}$, and $\chi_{\mathrm{cc}}$ for the ${ }^{14} \mathrm{~N}_{\mathrm{a}}$ and ${ }^{14} \mathrm{~N}_{\mathrm{i}}$ nuclei. The final rotational and quadrupole coupling constants are collected in the first column of Table II.

During the analysis of the rotational spectrum using the enriched sample ${ }^{15} \mathrm{~N}_{\mathrm{i}^{-}}{ }^{14} \mathrm{~N}_{\mathrm{a}}$ of $\operatorname{Trp}$ a set of weaker transitions with a characteristic pattern of $\mu_{\mathrm{a}}$-type R-branch transitions of a rotor close to a prolate asymmetric top was located, leading to the assignment of a new conformer. All the observed transitions exhibited quadrupole hyperfine splittings corresponding to a molecule containing one ${ }^{14} \mathrm{~N}$ nucleus (Table S4 of the supplementary material ${ }^{37}$ ) and were fitted using the same Hamiltonian described above. The resulting rotational and quadrupole coupling constants are given in the first column of Table III. The rotational constants and the spectroscopic selection rules for this rotamer are initially consistent with those predicted for conformers $\mathbf{I c + 2}, \mathbf{I c}+\mathbf{3}, \mathbf{I I c + 1}$, and IIc +2 showed in Table IV. As stated before, a conclusive identification cannot be reached only on this basis. The distinct orientation of these $\mathrm{NH}_{2}$ groups in these conformers causes a huge effect on the values of the quadrupole coupling constants $\chi_{\mathrm{aa}}, \chi_{\mathrm{bb}}$, and $\chi_{\mathrm{cc}}$ (see Table IV). Hence, a final comparison between the experimental and theoretical values of the quadrupole coupling constants (Tables III and IV, respectively) serves to discriminate between these conformers and allows the unequivocal identification of the observed rotamer as conformer IIc+1. This conformer has never been reported in any previous studies of Trp, ${ }^{3,22-25}$ although IIc+2 conformer - structurally very similar to IIc+1 - has been proposed. ${ }^{23,28}$ It seems that the spectral signatures of conformer $\mathbf{I I c + 1}$ has been misleadingly attributed to the existence of the conformer IIc+ $\mathbf{2}$, based on the relative 
TABLE III. Rotational parameters for the observed rotamer II of Trp.

\begin{tabular}{lcc}
\hline & ${ }^{15} \mathrm{~N}_{\mathrm{i}^{-}}{ }^{14} \mathrm{~N}_{\mathrm{a}}$ & ${ }^{15} \mathrm{~N}_{\mathrm{i}^{-}}{ }^{15} \mathrm{~N}_{\mathrm{a}}$ \\
\hline $\mathrm{A}^{\mathrm{a}}$ & $1281.310(15)^{\mathrm{b}}$ & $1272.455(8)$ \\
$\mathrm{B}$ & $333.70096(14)$ & $332.40803(8)$ \\
$\mathrm{C}$ & $287.10022(17)$ & $286.33178(9)$ \\
$\widehat{\vartheta}_{\mathrm{J}}$ & $0.01048(96)$ & $0.00945(44)$ \\
$\mathrm{N}_{\mathrm{a}}$ & & \\
$\chi_{\mathrm{aa}}$ & $-2.33(18)$ & $\ldots$ \\
$\chi_{\mathrm{bb}}$ & $1.95(12)$ & $\ldots$ \\
$\chi_{\mathrm{cc}}$ & $0.38(12)$ & $\ldots$ \\
$\chi_{\mathrm{cc}-} \chi_{\mathrm{cc}}$ & $1.573(62)$ & 10 \\
$\mathrm{~N}$ & 31 & 0.9 \\
$\sigma$ & 2.8 & $\ldots$ \\
\hline \hline
\end{tabular}

$\overline{{ }^{a} \mathrm{~A}, \mathrm{~B}, \mathrm{C} \text { are the rotational constants (in } \mathrm{MHz} \text { ); } \mathrm{J} \text { is the quartic centrifugal distortion }}$ constant (in $\mathrm{kHz}$ ); $\chi_{\mathrm{aa}}, \chi_{\mathrm{bb}}, \chi_{\mathrm{cc}}$ are the ${ }^{14} \mathrm{~N}$ nuclear quadrupole coupling constants (in $\mathrm{MHz}$ ); $\mathrm{N}$ is the number of fitted transitions; $\sigma$ is the rms deviation of the fit (in $\mathrm{kHz}$ ).

${ }^{\mathrm{b}} \mathrm{Standard}$ error in parentheses in units of the last digit.

energies $^{23}$ and conformational interconversion processes. ${ }^{28}$ It should be noted that our assignments are based on dozens of independent data for each species which provide a set of accurate spectroscopic parameters that are directly correlated with those obtained $a b$ initio and extremely sensitive to molecular structure. Our measurements offer an unmatched means to achieve an unambiguous identification of each observed conformer, avoiding any degree of ambiguity in the interpretation of the data.

A final effort to identify other conformers of Trp was undertaken using an enriched sample of ${ }^{15} \mathrm{~N}_{\mathrm{i}^{-}}{ }^{15} \mathrm{~N}_{\mathrm{a}}$ Trp. As we mentioned before, the presence of two quadrupolar nuclei splits each rotational transition into several hyperfine components thus reducing the intensity of the spectrum. ${ }^{15} \mathrm{~N}$ samples simplify the rotational spectra-by avoiding hyperfine effects, and thus help in the conformational searches. Detailed scans were performed looking for other lower energy conformers of Trp and some unidentified transitions were observed, but unfortunately no new assignments could be attained. Rotational spectra of the ${ }^{15} \mathrm{~N}_{\mathrm{i}^{-}}{ }^{15} \mathrm{~N}_{\mathrm{a}}$ isotopologues of conformers IIb+ and IIc+1 were predicted and readily detected at the expected frequency shifts. The measured transitions (Tables S5 and S6 of the supplementary material ${ }^{37}$ ) were fitted using Watson's semirigid rotor Hamiltonian $H_{\mathrm{R}}{ }^{(\mathrm{A})}$. The experimental rotational constants are given in the last columns of Tables II and III for conformers IIb+ and IIc+1, respectively. Relative intensity measurements were carried out, on different non-splitted $\mu_{\mathrm{a}}$-type measured rotational transitions of the ${ }^{15} \mathrm{~N}_{\mathrm{i}^{-}}{ }^{15} \mathrm{~N}_{\mathrm{a}}$ species of both conformers, and were used to estimate their relative populations in the supersonic jet. ${ }^{33}$ The results show that conformer IIb+ is approximately four times more abundant than conformer IIc+1 which is consistent with the predicted values of free energies (see Tables I and IV).

The 3D structures of the detected conformers are shown in Figure 2. They have been taken from those predicted by $a b$ initio calculations, based on the good agreement between the experimental and theoretical values of rotational constants. In addition, the ${ }^{14} \mathrm{~N}_{\mathrm{a}}$ nuclear quadrupole coupling constants allow us to establish, unequivocally, the orientation of the $\mathrm{NH}_{2}$ group within the molecule, and thus establish the intramolecular interactions in which this functional group is involved. Both conformers are stabilized by an $\mathrm{O}-\mathrm{H} \cdot \cdot \cdot \mathrm{N}$ hydrogen bond in an $\alpha-\mathrm{COOH}$ trans configuration (type II of the $\alpha$ amino acids ${ }^{34}$ ). Moreover, one of the hydrogen atoms of the amino group is pointing towards the $\pi$ electron density of the indole ring, indicating the existence of a $\mathrm{N}-\mathrm{H} \cdot \cdot \pi$ interaction. Both intramolecular hydrogen bonds form a chain that reinforces their strength through cooperative effects.

TABLE IV. Calculated ${ }^{\mathrm{a}}$ spectroscopic parameters for the $\mathbf{I c + 2}$, Ic+3, IIc+1, and $\mathbf{I I c}+\mathbf{2}$ conformers of Trp (extracted from Table S1 of the supplementary material ${ }^{37}$ ).

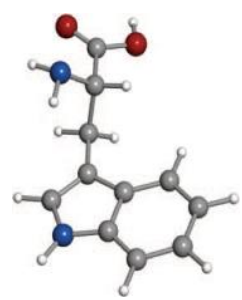

$\mathrm{Ic}+2$

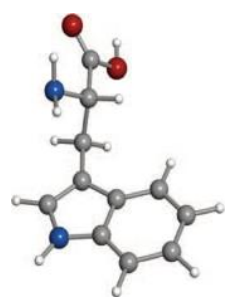

Ic+3

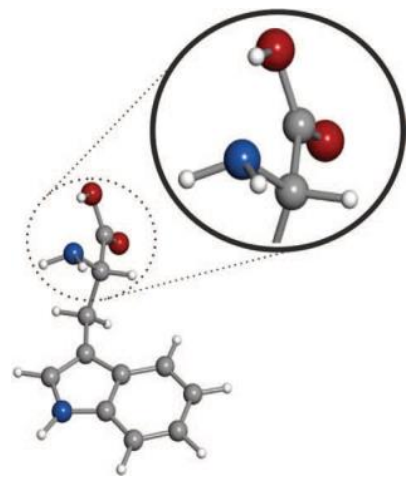

IIc + 1

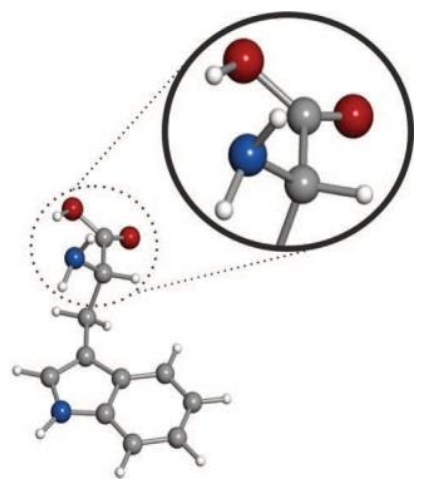

$\mathrm{IIc}+2$

\begin{tabular}{lcc}
\hline $\mathrm{A} / \mathrm{B} / \mathrm{C}^{\mathrm{b}}$ & $1247 / 342 / 299$ & $1253 / 336 / 292$ \\
$\chi_{\mathrm{aa}} / \chi_{\mathrm{bb}} / \chi_{\mathrm{cc}}\left(\mathrm{N}_{\mathrm{a}}\right)$ & $1.21 / 1.13 /-2.35$ & $1.27 / 1.75 /-3.02$ \\
$\left|\mu_{\mathrm{a}} / / \mu_{\mathrm{b}}\right| /\left|\mu_{\mathrm{c}}\right|$ & $2.5 / 1.7 / 1.1$ & $2.1 / 0.6 / 0.1$ \\
$\diamond \mathrm{E}$ & 949 & 1020 \\
$\mathrm{G}$ & 414 & 448
\end{tabular}

$1273 / 338 / 290$

$-2.42 / 0.20 / 2.23$

$5.3 / 4.2 / 2.2$

513

84

aptimized structures at the MP2/6-311++G(d,p) level

${ }^{\mathrm{b}} \mathrm{A}, \mathrm{B}, \mathrm{C}$ are the rotational constants (in MHz) for the isotopic species ${ }^{15} \mathrm{~N}_{\mathrm{i}-}{ }^{14} \mathrm{~N}_{\mathrm{a}} ; \chi_{\mathrm{aa}}, \chi_{\mathrm{bb}}, \chi_{\mathrm{cc}}$ are the ${ }^{14} \mathrm{~N}$ nuclear quadrupole coupling constants (in $\mathrm{MHz}$ ); $\mu_{\mathrm{a}}, \mu_{\mathrm{b}}, \mu_{\mathrm{c}}$ are the electric

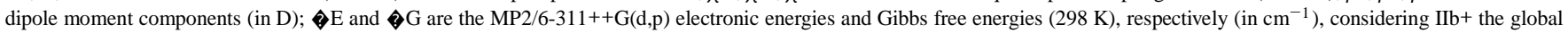
minimum. 


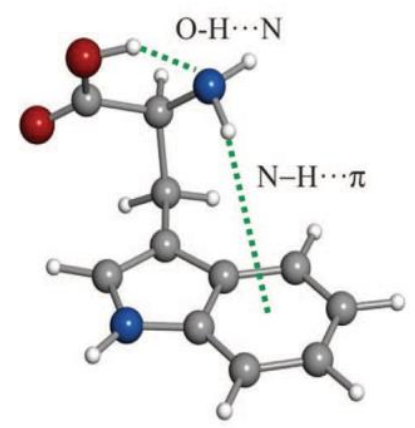

Rotamer I / Conformer IIb+

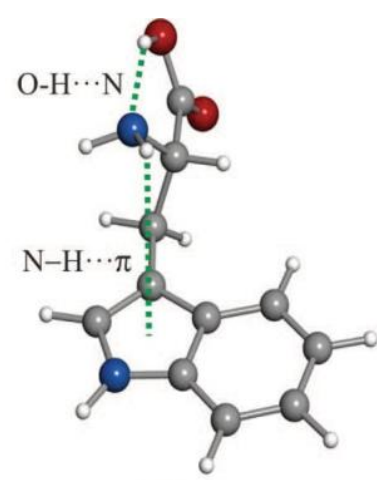

Rotamer II / Conformer IIc+1
FIG. 2. 3D structures of IIb + and IIc +1 conformers of Trp showing the intramolecular interactions which stabilize both structures.

\section{CONCLUSIONS}

The results presented in this paper detail the success of the LA-MB-FTMW technique in the study of the rotational spectrum of a heavy and complex biomolecule such as the amino acid Trp. In the present work, it was possible to bring solid tryptophan into the gas phase by laser ablation, minimizing the effects of photofragmentation, allowing a microwave spectroscopic study.

The role of the quadrupole coupling constants has been found to be priceless to identify without doubt the observed rotamers. The ${ }^{14} \mathrm{~N}$ nuclear quadrupole coupling constants also informs on the orientation of the side chain $\mathrm{NH}_{2}$ group with respect to the molecular frame and is relevant to extract conclusions about the intramolecular interactions that take place between the different functional groups.

The conformational behaviour observed for Trp shifts from the trend observed for aliphatic amino acids, where type $\mathrm{I}(\mathrm{N}-\mathrm{H} \cdot \cdot \mathrm{O})$ conformers are preferred as a global minimum. ${ }^{16,17,19,34}$ The two observed conformers for Trp are stabilized by an $\mathrm{O}-\mathrm{H} \cdot \cdots \mathrm{N}$ (type II) interaction between the carboxylic and amino groups of the side chain and a $\mathrm{N}-\mathrm{H} \cdot \cdots \pi$ interaction between the amino $\mathrm{NH}_{2}$ group and the high $\pi$ density sites of the indole ring. This conformational behaviour follows that of the aromatic amino acid phenylalanine $^{18}$ where the $\mathrm{N}-\mathrm{H} \cdot \cdots \pi$ interaction forms a chain with the $\mathrm{O}-\mathrm{H} \cdot \cdot \mathrm{N}$ interaction reinforcing the strength of hydrogen bonds through cooperative effects. ${ }^{35,36}$

\section{ACKNOWLEDGMENTS}

This work has been supported by the Ministerio de Ciencia e Innovación (Grant Nos. CTQ2010-19008 and Consolider-Ingenio 2010 CSD2009-00038) and the Junta de Castilla y León (Grant No. VA175U13). C.C. thanks the Junta de Castilla y León for a postdoctoral contract (Grant No. CIP13/01).

\footnotetext{
${ }^{1}$ K. Tanaka, H. Waki, Y. Ido, S. Akita, Y. Yoshida, and T. Yoshida, Rapid Commun. Mass Spectrom. 2, 151 (1988).

${ }^{2}$ F. Hillenkamp, M. Karas, R. C. Beavis, and B. T. Chait, Anal. Chem. 63 , 1193A-1201A(1991).

${ }^{3}$ T. R. Rizzo, Y. D. Park, L. A. Petenau, and D. H. Levy, J. Chem. Phys. 84, 2534 (1986).
}

${ }^{4}$ R. J. Levis, Annu. Rev. Phys. Chem. 45, 483 (1994).

${ }^{5}$ J. B. Fenn, M. Mann, C. K. Meng, S. F. Wong, and C. M. Whitehouse, Science 246, 64 (1989); Mass Spectrom. Rev. 9, 37 (1990).

${ }^{6}$ E. G. Robertson and J. P. Simons, Phys. Chem. Chem. Phys. 3, 1 (2001).

${ }^{7}$ B. C. Dian, J. R. Clarkson, and T. S. Zwier, Science 303, 1169 (2004).

${ }^{8}$ N. C. Polfer, B. Paizs, L. C. Snoek, I. Compagnon, S. Suhai, G. Meijer, G. von Helden, and J. Oomens, J. Am. Chem. Soc. 127, 8571 (2005).

${ }^{9}$ E. Gloaguen, B. de Courcy, J.-P. Piquemal, J. Pilmé, O. Parisel, R. Pollet, H. S. Biswal, F. Piuzzi, B. Tardivel, M. Broquier, and M. Mons, J. Am. Chem. Soc. 132, 11860 (2010).

${ }^{10}$ N. S. Nagornova, T. R. Rizzo, and O. V. Boyarkin, Science 336, 320 (2012).

${ }^{11}$ F. J. Lovas, Y. Kawashima, J. U. Grabow, R. D. Suenram, G. T. Fraser, and E. Hirota, Astrophys. J. 455, L201 (1995).

${ }^{12}$ U. Kretschmer, D. Consalvo, A. Knaack, W. Schade, W. Stahl, and H. Dreizler, Mol. Phys. 87, 1159 (1996).

${ }^{13}$ S. Antolínez, A. Lesarri, S. Mata, S. Blanco, J. C. López, and J. L. Alonso, J. Mol. Struct. 612, 125 (2002); A. Lesarri, S. Mata, J. C. López, and J. L. Alonso, Rev. Sci. Instrum. 74, 4799 (2003); A. Lesarri, S. Mata, S. Blanco, J. C. López, and J. L. Alonso, J. Chem. Phys. 120, 6191 (2004).

${ }^{14}$ R. D. Brown, P. D. Godfrey, J. W. V. Storey, and M. P. Bassez, J. Chem. Soc. Chem. Commun. 547-548 (1978); R. D. Suenram and F. J. Lovas, J. Mol. Spectrosc. 72, 372 (1978); J. Am. Chem. Soc. 102, 7180 (1980); S. J. McGlone, P. S. Elmes, R. D. Brown, and P. D. Godfrey, J. Mol. Struct. 485-486, 225 (1999).

${ }^{15}$ P. D. Godfrey, S. Firth, L. D. Hatherley, R. D. Brown, and A. P. Pierlot, J. Am. Chem. Soc. 115, 9687 (1993).

${ }^{16}$ J. L. Alonso, C. Pérez, M. E. Sanz, J. C. López, and S. Blanco, Phys. Chem. Chem. Phys. 11, 617 (2009), and references therein.

${ }^{17}$ M. E. Sanz, J. C. López, and J. L. Alonso, Phys. Chem. Chem. Phys. 12, 3573 (2010).

${ }^{18}$ C. Pérez, S. Mata, S. Blanco, J. C. López, and J. L. Alonso, J. Phys. Chem. A 115, 9653 (2011).

${ }^{19}$ I. Peña, M. E. Sanz, J. C. López, and J. L. Alonso, J. Am. Chem. Soc. 134, 2305 (2012).

${ }^{20}$ C. Cabezas, M. Varela, I. Peña, S. Mata, J. C. López, and J. L. Alonso, Chem. Commun. 48, 5934 (2012).

${ }^{21}$ M. E. Sanz, V. Cortijo, W. Caminati, J. C. López, and J. L. Alonso, Chem. Eur. J. 12, 2564 (2006).

${ }^{22}$ F. Piuzzi, I. Dimicoli, M. Mons, B. Tardivel, and Q. Zhao, Chem. Phys. Lett. 320, 282 (2000).

${ }^{23}$ L. C. Snoek, R. T. Kroemer, M. R. Hockridge, and J. P. Simons, Phys. Chem. Chem. Phys. 3, 1819 (2001).

${ }^{24}$ J. M. Bakker, L. M. Aleese, G. Meijer, and G. von Helden, Phys. Rev. Lett. 91, 203003 (2003)

${ }^{25}$ G. Rouille, M. Arold, A. Staicu, T. Henning, and F. Huisken, J. Phys. Chem. A 113, 8187 (2009).

${ }^{26}$ C. Bermúdez, S. Mata, C. Cabezas, and J. L. Alonso, "Tautomerism in Neutral Histidine?" (unpublished).

${ }^{27}$ Z. Huang and Z. Lin, J. Phys. Chem. A 109, 2656 (2005).

${ }^{28}$ A. Kaczor, I. D. Reva, L. M. Proniewicz, and R. Fausto, J. Phys. Chem. A 111, 2957 (2007).

${ }^{29}$ J. L. Alonso, V. Cortijo, S. Mata, C. Pérez, C. Cabezas, J. C. López, and W. Caminati, J. Mol. Spectrosc. 269, 41 (2011); C. Cabezas, M. Varela, I. Peña, J. C. López, and J. L. Alonso, Phys. Chem. Chem. Phys. 14, 13618 (2012).

${ }^{30}$ H. M. Pickett, J. Mol. Spectrosc. 148, 371 (1991).

${ }^{31}$ J. K. G. Watson, in Vibrational Spectra and Structure, edited by J. R. Durig (Elsevier, Amsterdam, 1977), Vol. 6, pp. 1-89.

${ }^{32}$ W. Gordy and R. L. Cook, Microwave Molecular Spectra, 3rd ed. (Wiley, New York, 1984).

${ }^{33}$ G. T. Fraser, R. D. Suenram, and C. L. Lugez, J. Phys. Chem. A 104, 11411146 (2000); S. Blanco, A. Lesarri, J. C. López, and J. L. Alonso, J. Am. Chem. Soc. 126, 11675-11683 (2004).

${ }^{34}$ S. Mata, V. Vaquero, C. Cabezas, I. Peña, C. Pérez, J. C. López, and J. L. Alonso, Phys. Chem. Chem. Phys. 11, 4141-4144 (2009).

${ }^{35}$ W. Saenger, Nature (London) 279, 343 (1979).

${ }^{36} \mathrm{G}$. A. Jeffrey, An Introduction to Hydrogen Bonding (Oxford University Press, New York, 1997). 